\title{
Photonic band-gap effect, localization, and waveguiding in the two-dimensional Penrose lattice
}

\author{
Mehmet Bayindir,* E. Cubukcu, I. Bulu, and E. Ozbay \\ Department of Physics, Bilkent University, Bilkent, 06533 Ankara, Turkey
}

(Received 9 February 2001; published 6 April 2001)

\begin{abstract}
We report experimental observation of a full photonic band gap in a two-dimensional Penrose lattice made of dielectric rods. Tightly confined defect modes having high quality factors were observed. Absence of the translational symmetry in Penrose lattice was used to change the defect frequency within the stop band. We also achieved the guiding and bending of electromagnetic waves through a row of missing rods. Propagation of photons along highly localized coupled-cavity modes was experimentally demonstrated and analyzed within the tight-binding approximation.
\end{abstract}

DOI: 10.1103/PhysRevB.63.161104

PACS number(s): 42.70.Qs, 42.60.Da, 61.44.Br, 71.15.Ap

Photonic crystals are artificial periodic structures in which the refractive index modulation gives rise to stop bands for electromagnetic waves (EM) within a certain frequency range in all directions. ${ }^{1,2}$ The existence of photonic band gap $^{3}$ and localized modes ${ }^{4}$ due to the Mie resonances ${ }^{5}$ and the Bragg scattering in these structures is of fundamental importance. Recently it was recognized that the photonic gaps can exist in two-dimensional (2D) quasicrystals. ${ }^{6-9}$ Defect characteristics in the same structures were also investigated theoretically ${ }^{10}$ and experimentally. ${ }^{11}$

A quasiperiodic system is characterized by a lack of longrange periodic translational order. But the quasiperiodic system has long-range band orientational order, so that it can be considered as an intermediate between periodic and random systems. ${ }^{12-14}$ Previously, one-dimensional versions of these structures, the Fibonacci lattices, were investigated. Existence of photonic stop bands ${ }^{15}$ and localization of light waves $^{16}$ in these one-dimensional photonic quasicrystals were reported. The Penrose tiles ${ }^{17}$ are composed of fat and skinny rhombic unit cells and fill the 2D plane nonperiodically as illustrated in Fig. 1. In electronic systems, localization phenomena in the 2D Penrose lattice was widely studied. ${ }^{18,19}$ Moreover, spectral gaps and localization were observed in 2D acoustical Penrose crystals. ${ }^{20,21}$ Recently, Krauss et al. demonstrated the diffraction pattern from a grating based on Penrose tiles. ${ }^{22}$

In this paper, we report on observation of the photonic band-gap effect in a 2D Penrose quasicrystal consisting of dielectric rods. Defect characteristics of various inequivalent sites of the crystal were investigated. It is observed that the EM waves can be guided and bended through the vacancy of removed rods along a line. We also measured transmission spectrum and dispersion relation of an array of coupled defects, and analyzed the experimental results within the classical wave analog of tight-binding approximation in solidstate physics. ${ }^{23}$

The 2D Penrose lattice was constructed by placing square shaped alumina rods, having refractive index 3.1 at the microwave frequencies and dimensions $0.32 \mathrm{~cm} \times 0.32 \mathrm{~cm}$ $\times 15.25 \mathrm{~cm}$, at each vertice of the skinny and fat rhombic cells (Fig. 1). The edge of each rhombus is $a=1.2 \mathrm{~cm}$. The experimental setup consists of a HP 8510C network analyzer and microwave horn antennas to measure the transmission- amplitude and transmission-phase spectra. The electric-field polarization vector of the incident EM field was parallel to the rods.

We first performed transmission measurements through the perfect Penrose crystal by varying angle of incidence of the EM waves (see the inset in Fig. 2). The crystal consisted of 236 rods, and had a square shape with dimensions $13 \mathrm{~cm} \times 13 \mathrm{~cm}$ corresponding to a filling fraction $\eta \sim 0.14$. As shown in Fig. 2, there is a strong attenuation, around 50 $\mathrm{dB}$, in transmission of EM waves through the crystal. The photonic band gap extends from 9.9 to $13.2 \mathrm{GHz}$. The same photonic band gap spectra were observed for different values of incidence angle between $0^{\circ}$ and $90^{\circ}$. The amount of power transmitted at conduction-band (upper band) frequencies was $20 \mathrm{~dB}$ less than the power transmitted at valenceband (lower band) frequencies. We performed the measurements up to $40 \mathrm{GHz}$, and we did not observe any other gaps in the transmission spectrum.

The defect characteristics of quasiperiodic photonic crystals can be different from the periodic case as pointed out in Ref. 6. Localization properties of the defect modes in quasicrystals depend on the position of the removed rod, since local environment of each site can be different from other sites. Therefore, we can get different defect frequencies within the band gap by removing rods from various posi-

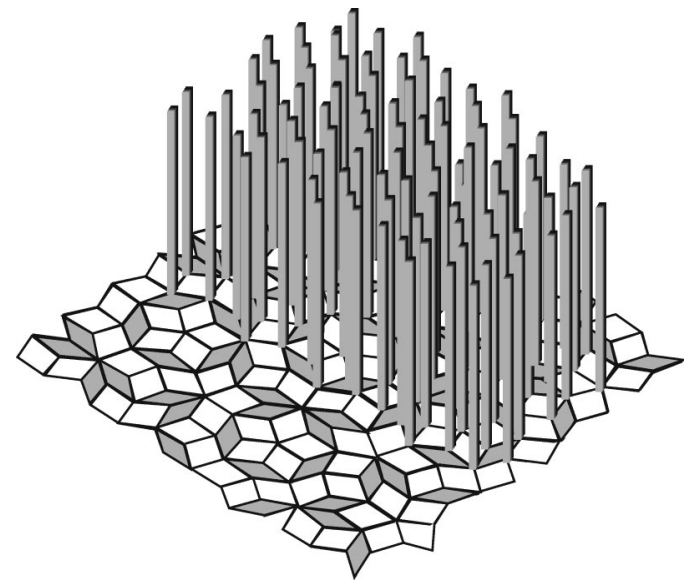

FIG. 1. Schematic drawing of a two-dimensional Penrose photonic crystal. The dielectric rods are placed at all vertices of fat and skinny rhombic cells. 


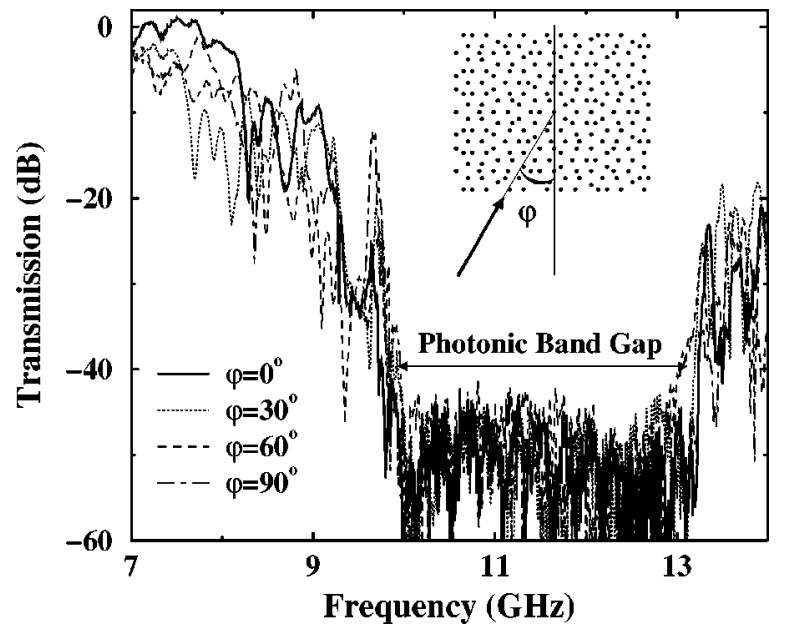

FIG. 2. Transmission characteristics of a Penrose dielectric crystal for various incidence angles. A stop band extending from 9.9 to 13.2 GHz was observed irrespective of the incidence angle. Inset: The (•) symbols denote vertices of the Penrose lattice.

tions. This feature might be important for certain applications. $^{24,25}$

We measured the transmission spectrum through a Penrose crystal, which consisted of 98 rods, with a single rod removed defect as labeled in the inset of Fig. 3. Each defect has different local properties, i.e., number and arrangement of neighboring rods. As shown in Fig. 3, we observed strongly localized cavity modes within the stop band of the crystal. The corresponding defect frequencies were $f_{A}$ $=11.436 \mathrm{GHz}, f_{B}=11.301 \mathrm{GHz}$, and $f_{C}=10.679 \mathrm{GHz}$. The quality factors, defined as center frequency divided by the peak's full width at half maximum, of these cavities were measured to be $Q_{A}=817, Q_{B}=513$, and $Q_{C}=305$.

Guiding and bending of EM waves in 2D periodic photonic crystals were theoretically demonstrated by Mekis

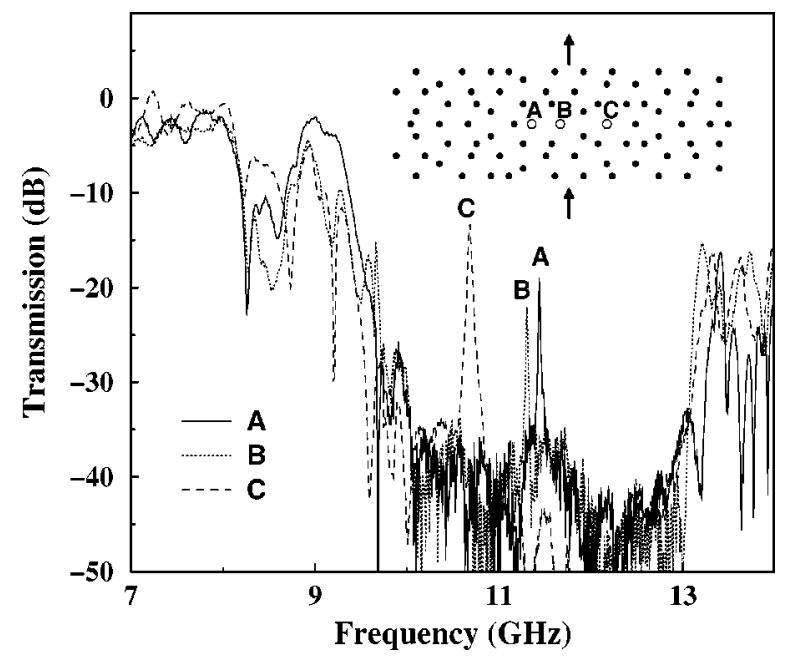

FIG. 3. Defect characteristics of a Penrose crystal consisting of 98 dielectric rods obtained by removing a single rod from various locations. Highly localized defect modes were observed. Since the crystal has many inequivalent defect sites, we can change the defect frequency within the stop band. The inset shows location of the defects. Here, point $B$ is positioned at the center of the crystal.

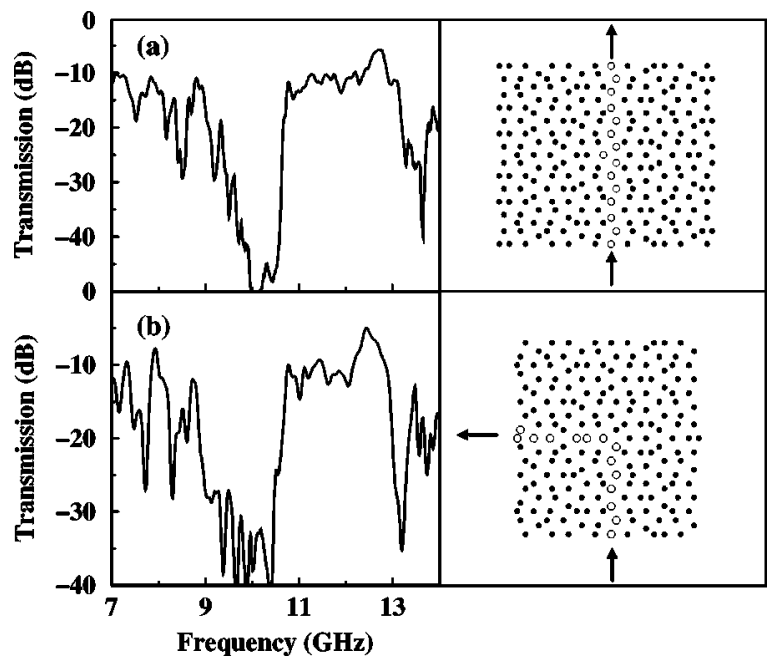

FIG. 4. (a) Waveguiding through a straight waveguide which was constructed by removing one row of rods ( $\bigcirc$ symbols) from the perfect Penrose crystal. (b) Bending of EM wave through a $90^{\circ}$ sharp bend. The right panels show schematics of the straight and bended waveguides.

et $a .^{26}$ and observed experimentally by Lin and co-workers. ${ }^{27}$ These phenomena was recently reported in $2 \mathrm{D}$ octagonal quasicrystals. ${ }^{7,10}$ In order to demonstrate the guiding and bending of the EM waves, we removed a row of rods from the Penrose lattice (see right panels in Fig. 4 for waveguide geometries). The transmission spectra corresponding to a straight (upper-right panel) and $90^{\circ}$ bended (lower-right panel) waveguides were measured and displayed in Figs. 4(a) and 4(b), respectively. For the straight waveguide, we observed guiding of EM waves from 10.60 to $13.22 \mathrm{GHz}$. For the bended waveguide, we achieved bending of EM waves from 10.60 to $13.06 \mathrm{GHz}$. The underlying physics of such waveguides can be investigated within the slab waveguide model, where the both crystals around the array of missing rods act as mirrors. Therefore, we expect that the EM wave to be guided through the introduced gap starting from a cutoff frequency, resonant frequency of the FabryPerot cavity, which depends on the width of the gap. ${ }^{28}$

Recently, we have reported a new type of propagation mechanism through an array of coupled cavities in onedimensional structures, ${ }^{29}$ and three-dimensional layer-bylayer photonic crystals. ${ }^{30,31}$ This mechanism depends on the coupling of each localized cavity mode with its neighbors, and therefore the EM energy can be transferred along the defect sites. In a periodic system, local properties of defect sites are the same, and we can represent each localized defect mode by a single function $\mathbf{E}_{\Omega}(\mathbf{r})$. Therefore, the waveguiding mode along the coupled-cavity array satisfies the Bloch condition. Can we observe this type of waveguiding in a quasiperiodic system? To demonstrate these phenomena, we looked for a path on the Penrose lattice along which all defect sites were identical. Such a path is illustrated in Fig. 5 (right panel, dotted line). There is a localized EM mode around each missing rod that is surrounded by seven neighboring rods.

We measured the transmission of EM waves through this 


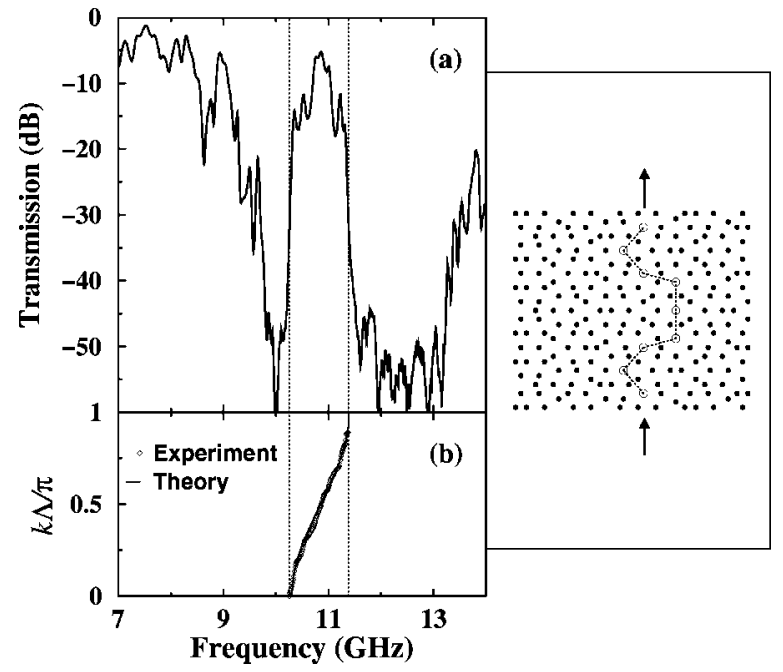

FIG. 5. (a) Transmission of EM waves through an array of coupled cavities which is shown in the right panel. The $\bigcirc$ symbols represent the missing rods. Photons can propagate along these localized coupled defect modes throughout a waveguiding band extending from 10.25 to $11.38 \mathrm{GHz}$. (b) Measured ( $\diamond$ symbols) and calculated (solid line) dispersion relations. The experimental result is in good agreement with the tight-binding prediction.

coupled-cavity array, that consisted of nine defect sites [Fig. 5(a)]. We observed that a defect band, waveguiding band, was formed within the photonic band gap. The guiding band extended from 10.25 to $11.38 \mathrm{GHz}$. The maximum transmission in the waveguiding band did not reach unity in contrast to what we had observed in $3 \mathrm{D}$ photonic crystals. ${ }^{30,31}$ This can be explained by the absence of confinement of the EM waves along the vertical direction (along the rod axis), ${ }^{32}$ that resulted in leakage of EM waves along this direction.

The dispersion relation of the coupled-cavity structure can be obtained from transmission-phase measurements. ${ }^{31,33,34}$ By using the net phase difference $\delta \phi$ between the phase of the EM wave propagating through the photonic crystal and the phase of the EM wave propagating in free space, we can determine the wave vector $k$ of the crystal as a function of frequency $\omega$ from

$$
k \Lambda=\delta \phi \Lambda / L+2 \pi \omega \Lambda / c,
$$

where $c$ is the speed of the light, $\Lambda=2.28 \mathrm{~cm}$ is the distance between two consecutive defects, and $L=20.6 \mathrm{~cm}$ is the to- tal crystal thickness.

The dispersion relation can also be determined within the TB approximation ${ }^{31}$ which is given by

$$
\omega_{k} \simeq \Omega[1+\kappa \cos (k \Lambda)],
$$

where $\Omega=10.85 \mathrm{GHz}$ is the resonance frequency corresponding to a single defect, $\kappa \sim-0.05$ is a TB parameter that was experimentally determined from the splitting of two coupled cavities and the width of waveguiding band. ${ }^{30}$ Figure 5(b) shows the comparison of the measured ( $\diamond$ symbols) and the calculated (solid line) dispersion relations. The good agreement between the experiment and the theory is an indication of the usefulness of the TB scheme in quasiperiodic photonic structures.

At this point it is natural to ask the following question. Can we observe photonic band gaps and the waveguiding phenomena in the absence of periodicity? The answer is yes. We have observed that the photonic band gap and metallicity gap persist even if large amount of positional disorder was introduced in 2D dielectric and metallic photonic crystals, respectively. It was also observed that the defect frequency of a single rod removed cavity can be tuned by changing position of the defect sites in weakly disordered 2D dielectric and metallic photonic crystals. Waveguiding was also achieved for weakly disordered systems. ${ }^{35}$

In conclusion, we have experimentally observed that the EM waves cannot propagate within a certain frequency range through a $2 \mathrm{D}$ quasicrystal that was composed of the Penrose tiling of dielectric rods. It was demonstrated that by removing a single rod from an otherwise perfect Penrose lattice, we could create a highly localized cavity mode. Since the crystal had many inequivalent sites, we achieved different defect frequencies within the photonic band gap. Moreover, we demonstrated guiding and bending of the EM waves along a row of missing rods. Particular attention was devoted to the transferring of the EM energy through a coupled-cavity array. Using a theory based on the tight-binding approximation, we obtained good agreement between the measured and the calculated dispersion relations.

This work was supported by NATO Grant No. SfP971970, National Science Foundation Grant No. INT9820646, Turkish Department of Defense Grant No. KOBRA-001, and Thales JP8.04.
*Author to whom correspondence addressed. Electronic address: bayindir@fen.bilkent.edu.tr

${ }^{1}$ J. D. Joannopoulos, R. D. Meade, and J. N. Winn, Photonic Crystals: Molding the Flow of Light (Princeton University Press, Princeton, NJ, 1995).

${ }^{2}$ For a recent review, see articles in Photonic Band Gap Materials, edited by C. M. Soukoulis (Kluwer, Dortrecht, 1996).

${ }^{3}$ E. Yablonovitch, Phys. Rev. Lett. 58, 2059 (1987).

${ }^{4}$ S. John, Phys. Rev. Lett. 58, 2486 (1987).

${ }^{5}$ C. F. Bohren and D. R. Huffman, Absorption and Scattering of Light by Small Particles (Wiley, New York, 1983), Chap. 4.
${ }^{6}$ Y. S. Chan, C. T. Chan, and Z. Y. Liu, Phys. Rev. Lett. 80, 956 (1998).

${ }^{7}$ C. Jin, B. Cheng, B. Man, Z. Li, and D. Zhang, Appl. Phys. Lett. 75, 1848 (1999).

${ }^{8}$ M. E. Zoorob, M. D. B. Charlton, G. J. Parker, J. J. Baumberg, and M. C. Netti, Nature (London) 404, 740 (2000).

${ }^{9}$ X. Zhang, Zhao-Quing Zhang, and C. T. Chan, Phys. Rev. B 63, 081105(R) (2001).

${ }^{10}$ S. S. M. Cheng, Lie-Ming Li, C. T. Chan, and Z. Q. Zhang, Phys. Rev. B 59, 4091 (1999).

${ }^{11}$ C. Jin, B. Cheng, B. Man, Z. Li, and D. Zhang, Phys. Rev. B 61, 
10762 (2000).

${ }^{12}$ D. Levine and P. J. Steinhardt, Phys. Rev. B 34, 596 (1986).

${ }^{13}$ P. J. Steinhardt and S. Ostlund, The Physics of Quasicrystals (World Scientific, Singapore, 1987).

${ }^{14}$ C. Janot, Quasicrystals: A Primer (Clarendon Press, Oxford, 1994).

${ }^{15}$ T. Hattori, N. Tsurumachi, S. Kawato, and H. Nakatsuka, Phys. Rev. B 50, R4220 (1994).

${ }^{16}$ W. Gellermann, M. Kohmoto, B. Sutherland, and P. C. Taylor, Phys. Rev. Lett. 72, 633 (1994).

${ }^{17}$ R. Penrose, Bull. Inst. Math. Appl. 10, 266 (1974); M. Gardner, Sci. Am. 236, 110 (1977).

${ }^{18}$ M. Kohmoto and B. Sutherland, Phys. Rev. Lett. 56, 2740 (1986).

${ }^{19}$ J. Vidal, R. Mosseri, and B. Doucot, Phys. Rev. Lett. 81, 5888 (1998).

${ }^{20}$ S. He and J. D. Maynard, Phys. Rev. Lett. 62, 1888 (1989).

${ }^{21}$ F. M. de Espinosa and M. Torres, Appl. Phys. Lett. 65, 1352 (1994).

${ }^{22}$ T. F. Krauss and R. M. De La Rue, Prog. Quantum Electron. 23, 51 (1999).

${ }^{23}$ N. W. Ashcroft and N. D. Mermin, Solid State Physics (Saunders, Philadelphia, 1976), p. 175.
${ }^{24}$ P. R. Villeneuve, D. S. Abrams, S. Fan, and J. D. Joannopoulos, Opt. Lett. 21, 2017 (1996); P. R. Villeneuve, S. Fan, and J. D. Joannopoulos, Phys. Rev. B 54, 7837 (1996).

${ }^{25}$ S. Noda, A. Chutinan, and M. Imada, Nature (London) 407, 608 (2000).

${ }^{26}$ A. Mekis et al., Phys. Rev. Lett. 77, 3787 (1996).

${ }^{27}$ Shawn-Yu Lin, E. Chow, V. Hietala, P. R. Villeneuve, and J. D. Joannopoulos, Science 282, 274 (1998).

${ }^{28}$ B. Temelkuran and E. Ozbay, Appl. Phys. Lett. 74, 486 (1999).

${ }^{29}$ Mehmet Bayindir, S. Tanriseven, and E. Ozbay, Appl. Phys. A: Mater. Sci. Process. 72, 117 (2001).

${ }^{30}$ Mehmet Bayindir, B. Temelkuran, and E. Ozbay, Phys. Rev. Lett. 84, 2140 (2000), and references therein.

${ }^{31}$ Mehmet Bayindir, B. Temelkuran, and E. Ozbay, Phys. Rev. B 61, R11 855 (2000)

${ }^{32}$ S. G. Johnson, P. R. Villeneuve, S. Fan, and J. D. Joannopoulos, Phys. Rev. B 62, 8212 (2000).

${ }^{33}$ W. M. Robertson et al., Phys. Rev. Lett. 68, 2023 (1992).

${ }^{34}$ E. Ozbay et al., Opt. Lett. 19, 1155 (1994).

${ }^{35}$ Mehmet Bayindir, E. Cubukcu, I. Bulu, and E. Ozbay (unpublished). 